\title{
ON TORSION SUBGROUPS OF LIE GROUPS
}

\author{
DONG HOON LEE
}

\begin{abstract}
We are concerned with torsion subgroups of Lie groups. We extend the classical result of $\mathrm{C}$. Jordan on the structure of finite linear groups to torsion subgroups of connected Lie groups.
\end{abstract}

1. In [1], Boothby and Wang proved that, for any connected Lie group $G$, there exists a number $k(G)$ such that any finite subgroup contains an abelian normal subgroup whose index is bounded by $k(G)$, thereby generalizing the famous result of $\mathrm{C}$. Jordan for $\operatorname{GL}(n, C)$. Moreover, by using an integral formula of Weyl, they described the bound explicitly. The main purpose of this note is to extend their result to torsion subgroups of connected Lie groups by using the same bound $k(G)$ as above.

In order to describe our result, we first recall the bound of index $k(G)$ as presented in [1]. Let $G$ be a connected Lie group and $K$ a maximal compact subgroup of $G$. Let $\mathcal{K}$ denote the Lie algebras of $K$ and let $Q$ be the set consisting of all $X \in \mathcal{K}$ such that the absolute values of the eigenvalues of ad $X$ are all less than $\pi / 6$, and let $U=\exp _{K} Q$. Then $k(G)$ is defined to be $\mu(K) / \mu(U)$ where $\mu$ is an invariant Haar measure of $K$. That $k(G)$ does not depend on the choice of $\mu$ is clear. Then our main result states:

THEOREM. Let $G$ be a connected Lie group. Then every torsion subgroup of $G$ contains an abelian normal subgroup whose index is bounded by $k(G)$.

The following lemmas are needed for the proof of the theorem.

Lemma 1 (Selbert [2, p. 154]). Every finitely generated subgroup of $\mathrm{GL}(n, C)$ contains a normal torsion free subgroup of finite index.

LEMMA 2. Every torsion subgroup of a connected Lie group is contained in a maximal compact subgroup.

Proof. Let $H$ be a torsion subgroup of a connected Lie group $G$ and let $\mathcal{G}$ be its Lie algebra. Let Ad: $G \rightarrow \mathrm{GL}(\mathcal{G})$ be the adjoint representation of $G$ and let $\delta$ be the subalgebra of the associative algebra End(G) of all the endomorphisms of the linear space $\mathcal{G}$, which is generated by $\mathrm{Ad}(H)$. As $\mathcal{S}$ is finitedimensional, there exists a finitely generated subgroup $L$ of $H$ such that the algebra $\delta$ is generated by the subgroup $\operatorname{Ad}(L)$. By Lemma $1, \operatorname{Ad}(L)$ is finite. As ker Ad is the center of $G$, we see that $L$ is finite modulo its center. It follows then that $L$ itself is finite. Let $C$ be a maximal compact subgroup which contains $L$. We show that $H<C$. Indeed, let $x \in H$. Since Ad $(x)$ is contained in $\delta$ which is generated by $\operatorname{Ad}(L)$ and $\operatorname{Ad}(L)$ leaves invariant the Lie

Received by the editors March 21, 1975.

AMS (MOS) subject classifications (1970). Primary 22C05, 22E20; Secondary 20F50.

(c) American Mathematical Society 1976 
subalgebra $C$ corresponding to $C$, we see that $\operatorname{Ad}(x)$ leaves $C$ invariant. Thus $x$ normalizes $C$. Since $x$ is a torsion element, the maximality of $C$ implies that $x \in C$. Hence $H<C$.

The proof of the following lemma may be found in [1, p. 286].

LEMMA 3. Let $G$ be a compact Lie group with Lie algebra $\mathfrak{G}$ and let ad denote the adjoint representation of $\mathcal{G}$. For each $0<c<\pi$, let $Q_{c}$ denote the totality of $X \in \mathcal{G}$ such that all the eigenvalues of $\operatorname{ad} X$ have absolute value less than $c$. Let $U_{c}=\exp Q_{c}$. Then we have $U_{c}=U_{c}^{-1}, g U_{c} g^{-1}=U_{c}$ for all $g \in G$, and $U_{c} U_{c^{\prime}} \subseteq U_{c+c^{\prime}}$, whenever $0<c, c^{\prime}, c+c^{\prime}<\pi$.

Now we are ready to prove the announced theorem.

By Lemma 2, we may assume that $G$ itself is compact. In [1] it is shown that if $F$ is a finite subgroup of $G$, then $F \cap U_{\pi / 3}$ is commutative. We claim that $H \cap U_{\pi / 3}$ is commutative. In fact, if $x, y \in H \cap U_{\pi / 3}$, then, by Lemma 1, $\{\operatorname{Ad}(x), \operatorname{Ad}(y)\}$ generates a finite group, and hence $\{x, y\}$ generates a finite subgroup of $G$ and $x y=y x$ follows. Thus $H \cap U_{\pi / 3}$ generates an abelian subgroup. Let $M$ denote the closure of $H$ and let $B$ denote the closure of the subgroup generated by $H \cap U_{\pi / 3}$. Thus $B$ is a closed abelian subgroup of the compact Lie group $M$. We claim that $B \cap H$ is a normal subgroup of $H$ with finite index. In fact, the normality follows from the invariance of $U_{c}$ under conjugation (Lemma 3).

Let $M_{0}$ denote the identity component of $M$. Since $H$ is dense in $M$, we have $M \cap U_{\pi / 3} \subseteq \overline{H \cap U_{\pi / 3}} \subseteq B$, where, for any set $A, \bar{A}$ denotes the closure of $A$. Since $M \cap U_{\pi / 3}$ is a neighborhood of 1 in $M$, the subgroup generated by $M \cap U_{\pi / 3}$ is open in $M$, and hence contains $M_{0}$. Thus $M_{0} \leqslant B$ and we see that $[M: B] \stackrel{<}{<}$. Now $[H: H \cap B]=[H B: B] \leqslant[M: B]<\infty$, proving that $H$ $\cap B$ is of finite index in $H$. It remains to show that the abelian normal subgroup $H \cap B$ of $H$ has index less that $k(G)$. To do this, we proceed as in [1].

Let $A=H \cap B$ and let $m=[H: A]$. Let $h_{1} A, \ldots, h_{m} A$ be the complete listing of the distinct cosets of $A$ in $H$. Then the open sets $h_{1} U_{\pi / 6}, \ldots, h_{m} U_{\pi / 6}$ are pairwise disjoint. For otherwise $h_{i} U_{\pi / 6} \cap h_{j} U_{\pi / 6} \neq \varnothing$ for some $i \neq j$ which would imply that $h_{j}^{-1} h_{i} \in U_{\pi / 6} U_{\pi / 6} \subseteq U_{\pi / 3}^{\pi / 6} \cap H \subseteq B \cap H=A$. Thus $\mu(G) \geqslant \sum_{i} \mu\left(h_{i} U_{\pi / 6}\right)=m \mu\left(U_{\pi / 6}\right)$, proving that $[H: A]=m \leqslant k(G)$.

The following is immediate from Lemma 2.

Corollary. A discrete torsion subgroup of a connected Lie group is finite.

Using the main result, we prove the following:

Corollary. Let $G$ be a connected Lie group. Then $G$ is a toroid if and only if $G$ contains a dense torsion subgroup.

Proof. Clearly a toroid contains a dense torsion subgroup. Assume, conversely, that $G$ contains a dense torsion subgroup $H$. By the theorem, $H$ contains an abelian normal subgroup $A$ of finite index and $\bar{A}$ is compact by Lemma 2. Let $x_{1} A, \ldots, x_{m} A$ be the complete listing of the cosets of $A$ in $H$. Then $G=U_{i=1}^{m} \overline{x_{i} A}=U_{i=1}^{m} x_{i} \bar{A}$. Hence $\bar{A}$ is open and closed in $G$. Since $G$ is connected, it follows that $G=\bar{A}$ proving that $G$ is a toroid. 


\section{REFERENCES}

1. W. M. Boothby and H. C. Wang, On the finite subgroups of connected Lie groups, Comment. Math. Helv. 39 (1965), 281-294. MR 31 \#4856.

2. A. Selberg, On discontinuous groups in higher-dimensional symmetric spaces, Contributions to Function Theory (Internat. Colloq. Function Theory, Bombay, 1960), Tata Institute of Fundamental Research, Bombay, 1960, pp. 147-164. MR 24 \#188.

Department of Mathematics, Case Western Reserve University, Cleveland, Ohio 44106 